\title{
Endometrial cancer after pelvic radiotherapy: a case-report and review of literature
}

\author{
Sara Passos Silva ${ }^{1 *}$, Andreia Martins Miranda ${ }^{1}$, Elisabete Gonçalves ${ }^{1}$, Sónia Carvalho² \\ Ana Simas ${ }^{3}$, Agostinho Carvalho ${ }^{1}$
}

\begin{abstract}
${ }^{1}$ Department of Obstetrics and Gynaecology, ${ }^{2}$ Department of Anatomic Pathology, ${ }^{3}$ Department of Medical Oncology ULSAM, Viana do Castelo, Portugal
\end{abstract}

Received: 25 January 2022

Accepted: 10 February 2022

\author{
*Correspondence: \\ Dr. Sara Passos Silva, \\ E-mail: saracpsilva7@gmail.com
}

Copyright: () the author(s), publisher and licensee Medip Academy. This is an open-access article distributed under the terms of the Creative Commons Attribution Non-Commercial License, which permits unrestricted non-commercial use, distribution, and reproduction in any medium, provided the original work is properly cited.

\begin{abstract}
Cancer treatment has evolved with advances in radiation and chemotherapy. However, over time the effects of ionizing radiation become more evident. In spite of attempting a more targeted therapeutic approach, the harmful effects of radiation on healthy tissues are many times inevitable. We report the case of a young woman who underwent chemoradiotherapy for stage IB2 cervical cancer with no evidence of relapse during 10 years follow-up. Instead she had radiation associated colitis and cystitis and vertebral necrosis. After 10 years an endometrial cancer was diagnosed. Radiation associated endometrial cancer is rare and may present with atypical symptoms making the diagnosis challenging. High degree of suspicion is necessary for an on time diagnosis and treatment.
\end{abstract}

Keywords: Gynecology, cervical cancer, radiation-associated cancer; endometrial cancer

\section{INTRODUCTION}

Cervical cancer is the eighth most commonly diagnosed cancer and the ninth leading cause of cancer death in the world. ${ }^{1}$ Chemoradiotherapy has been the standard of care for patients with locally advanced disease for almost two decades. $^{2}$ But with longer survivorship rates after treatment, long term effects of pelvic irradiation become an important issue. Side effects of radiation therapy come from damage to healthy cells and tissues near the treatment area predisposing to inflammation and damage to adjacent structures and rarely a second tumor can appear. ${ }^{3}$ Studies pointed that after radiation therapy for cervical cancer patients are at a higher risk of developing poorly differentiated, high grade histological types of endometrial cancer that commonly present in advanced stages. ${ }^{3}$

\section{CASE REPORT}

A 35-year-old caucasian woman presented for a gynecologic routine examination in February 2009 and a $4 \mathrm{~cm}$ exofitic lesion was found in the uterine cervix. The biopsy revealed an invasive endocervical adenocarcinoma, usual type (Figure 1). No linfo-vascular invasion was found. The malignancy was staged IB2 according to the International federation of gynecology and obstetrics (FIGO- 2009). Concomitant external beam radiotherapy (EBRT) with $15 \mathrm{Mv}$ photons, weekly cisplatin $40 \mathrm{mg} / \mathrm{m}^{2}$ for five weeks and two brachytherapy applications were performed after multidisciplinary team decision at a reference cancer hospital. treatments ended in September 2010 with complete response. One year later, she initiated frequent episodes of hematuria and haematochezia in relation with radiation-induced proctitis and cystitis difficult to control even with hyperbaric oxygen therapy. 
In 2018 a vertebral necrosis radiotherapy-associated was diagnosed causing neuropathic pain in right lower leg and requiring multimodal analgesia. In December 2019, she presented to the emergency department complaining of pelvic pain, bladder tenesmus, vaginal bleeding and asthenia. Examination showed a vaginal shortening, vaginal cuff with no evidence of disease and a tender pelvic mass measuring about $10 \mathrm{~cm}$. She was admitted with a severe anemia. Urethral catheterization revealed frank hematuria and a bladder irrigation system was placed for clots removing. Computer tomography (CT) scan revealed a volumous hematometra $101 \times 70 \mathrm{~mm}$ with compression of adjacent structures. Aspirative biopsy was negative for malignancy. PET scan was done with no evidence of recurrence or other malignancy. Because of hemodynamic instability and enlargement of hematometra with the need of blood transfusions, poor clinical response to bladder irrigation system and electrocautery and hyperbaric oxygen treatment, histerectomy was proposed. At surgery, we found an uterus transformed in a $15 \mathrm{~cm}$ soft mass, radiogenic bladder without any distensibility and bilateral hydronephrosis. A hysterectomy with bilateral salpingoooforectomy was done. Histology revealed a serous uterine carcinoma invading less than one-half of the myometrium, Stage IA FIGO; pT1aNx. (Figure 2-3). Pathological review with biomolecular analysis ruled out a recurrence. She was proposed to adjuvant chemotherapy. Four months later due to maintaining severe bladder tenesmus a cystectomy was done. During surgery, peritoneal carcinomatosis was diagnosed with ascitic free fluid, multiple implants thought Douglas pouch, bladder and several in small bowell and peritoneum. Cystectomy, bilateral ureterostomy, peritoneal biopsies were performed (Figure 4). Histology confirmed implants with serous uterine carcinoma. She was proposed for palliative chemotherapy and referred for palliative care.

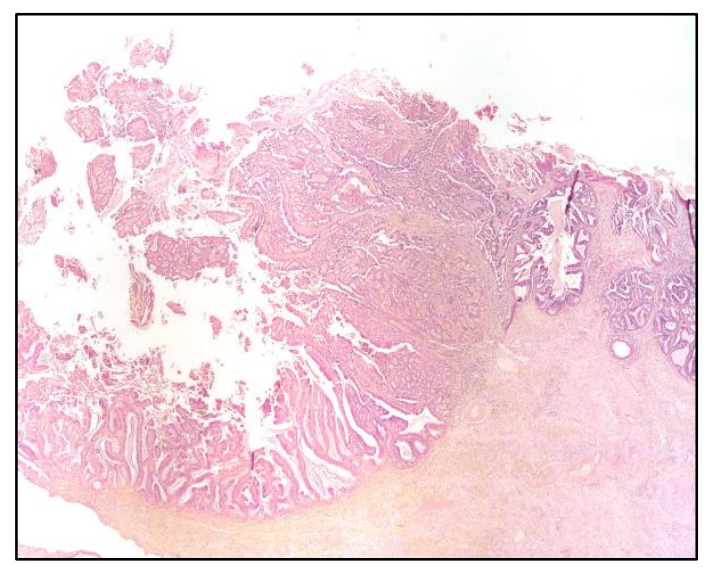

Figure 1: Cervical adenocarcinoma; HPV related, characterized by easily recognized glands lined by stratified columnar cells with eosinophilic cytoplasm and moderate nuclear pleomorphism. Synchronous adenocarcinoma "in situ" was present.

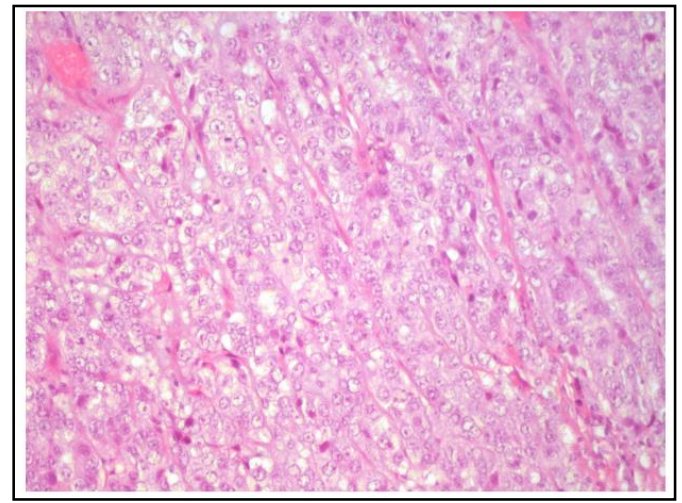

Figures 2: Endometrial serous carcinoma.

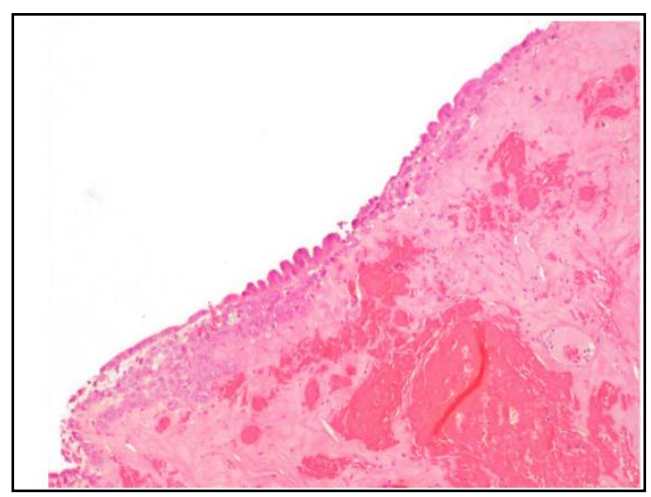

Figures 3: A section showing irregular endometrium with polypoid intracavitary projections characterized by solid nests and sheets of tumor cells showing marked nuclear pleomorphism. The background endometrium exhibited endometrial intraepithelial carcinoma. Tumor cells showed p53 strong and diffuse $(>75 \%)$ staining and HPV DNA was not detected in paraffin-embedded tumor samples.

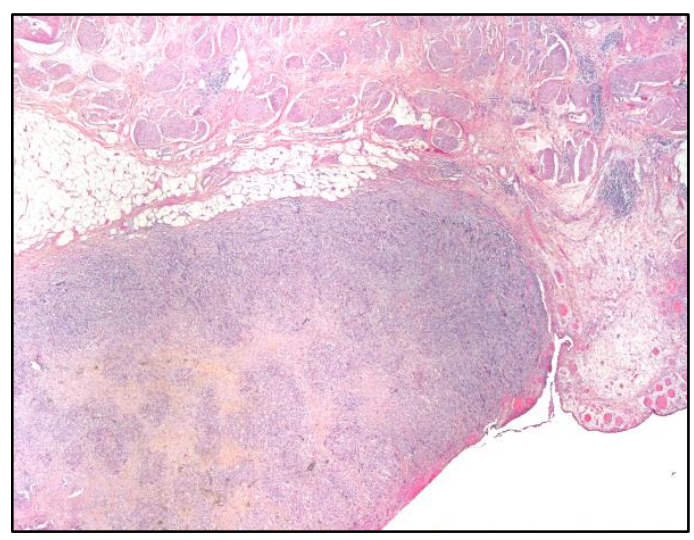

Figure 4: Metastasis of endometrial cancer; perivesical peritoneal nodule showing infiltrating serous carcinoma.

\section{DISCUSSION}

Endometrial cancer developing after irradiation of pelvic area has been called radiation endometrial cancer (REC) 
and represents a group of heterogeneous tumors, a late and rare complication of radiotherapy. REC has been suggested to be a high risk disease with poorly differentiated tumors, more local and distant spread and therefore with lower survival rates. ${ }^{4}$ The primary tumor is cervical cancer in the majority of written reports. The mean latency period between the diagnosis of first tumor and REC was 14 years, range from 5 to 28 years. ${ }^{3,4-8} \mathrm{~A}$ delay in the diagnosis of these tumors may explain why the disease presents in more advanced stages. Clinical presentation is often atypical with abdominal pain being the most common symptom whereas in sporadic types vaginal bleeding is almost always present. Just like we have seen in our patient, that never complaint about vaginal bleeding.

One explanation is the fact that a well-known complication of RT, stenosis and obliteration of cervical canal block the passage of blood. Keeping these in mind a fluid-filled endometrial cavity should raise the suspicion of endometrial cancer. In fact, Lopes et al in a 2018 manuscript highlight the importance of hematometra/hidrometra in the diagnosis of these endometrial cancers. In their review hematometra was observed in $85 \%$ of the sample, with large volumes in most cases. ${ }^{3}$ The threshold for an endometrial biopsy should be low. Lopes et al also reported that RM identified 10 in 11 patients with endometrial cancers. Image staging is challenging particularly local staging due to the heterogeneity, irregularity and size of lesions associated to the additional findings of hematometra and anatomical distortion of radiation. Drainage of hematometra prior to RM may be advised. The role CT scan is mainly in assessment of distant spread. ${ }^{3}$ Kwon et al described 8 patients with REC occurring 5 to 23 years, average of 13 years following radiotherapy for cervical cancer, and only 2 of them presented with vaginal bleeding. ${ }^{5}$

All the patients had locally invasive disease or distant metastases and only 1 had a histologic differentiated carcinoma. Pothuri et al also described 23 patients with REC occurring 6 to 27 years after, mean interval of 14 years. ${ }^{6}$ When comparing with sporadic cancers significant differences were noted in stage, grade, histologic subtypes and survival. The REC group with higher stages, grades, higher risk subtypes and lower survival rates. Patients with REC are poor candidates for radiotherapy because they have received it previously. Hysterectomy is difficult because of extensive fibrosis particularly in cervix resection, so the main treatment is chemotherapy. ${ }^{9}$ Our study supports previous literature in that radiation-associated metachronous uterine malignancy is associated with a worse survival compared to non-metachronous tumors and has described hematometra limited the utility of imaging in local staging.

\section{CONCLUSION}

Radiation endometrial cancers are rare but with a worse prognosis. The threshold for diagnostic work-up should be low in the presence of abdominal pain/discomfort or a hematometra imaging finding in a woman with a prior history of pelvic irradiation after a long latency of exposure. REC appears to be a unique entity in many aspects: presenting symptoms, diagnostic difficulty, extent of the cancer, and poor outcome. Clinician's awareness is essential for a timely diagnosis.

Funding: No funding sources

Conflict of interest: None declared

Ethical approval: Not required

\section{REFERENCES}

1. The Global Cancer Observatory - November, 2020. Available at: https://gco.iarc.fr/. Accessed on 20 November 2021.

2. Marth C, Landoni F, Mahner S, McCormack M, Gonzalez-Martin A, Colombo N; ESMO Guidelines Committee. Cervical cancer: ESMO Clinical Practice Guidelines for diagnosis, treatment and follow-up. Ann Oncol. 2017;28(4):72-83.

3. Lopes J, Horta M, Cunha TM, Endometrial cancer after radiation therapy for cervical carcinoma: a radiological approach. Eur J Radiol. 2018;25(3):459.

4. Kumar S, Shah JP, Bryant CS, Seward S, Ali-Fehmi R, Morris RT, et al. Radiation-associated endometrial cancer. Obstet Gynecol. 2009;113(1): 319-25.

5. Kwon TH, Prempree T, Tang CK, VillaSanta U, Scott RM. Adenocarcinoma of the uterine corpus following irradiation for cervical cancer. Gynecol Oncol. 1981;11(1):102-13.

6. Pothuri B, Ramondetta L, Eifel P, Deavers MT, Wilton A, Alektiar K, et al. Radiation-associated endometrial cancers are prognostically unfavorable tumors: a clinicopathologic comparison with 527 sporadic endometrial cancers. Gynecol Oncol. 2006;103(3):948-51.

7. Fehr PE, Prem KA. Malignancy of the uterine corpus following irradiation therapy for squamous cell carcinoma of the cervix. Am J Obstet Gynecol. 1974;119:685-92.

8. Rodriguez J, Hart WR. Endometrial cancer occurring 10 or more years after pelvic irradiation for carcinoma. Int J Gynecol Pathol. 1982;1:135-44.

9. Parkash V, Carcangiu ML. Uterine papillary serous carcinoma after radiation therapy for carcinoma of the cervix. Cancer. 1992;69(2):496-501.

Cite this article as: Silva SP, Miranda AM, Gonçalves E, Carvalho S, Simas A, Carvalho A. Endometrial cancer after pelvic radiotherapy: a casereport and review of literature. Int J Reprod Contracept Obstet Gynecol 2022;11:916-8. 